\title{
A STUDY ON THE EFFECTS OF AGOMELATINE ON FOOD INTAKE AND BODY WEIGHT IN RESTRAINT STRESS MODEL IN ADULT SWISS ALBINO MICE
}

\author{
SHANMUGAPRIYA S*, NIVEDHITHAA S, BHUVANESWARI K \\ Department of Pharmacology, PSG Institute of Medical Sciences and Research, Peelamedu, Coimbatore, Tamil Nadu, India. \\ Email: somasundaram999@rediffmail.com
}

Received: 01 May 2017, Revised and Accepted: 25 May 2017

\section{ABSTRACT}

Objectives: Agomelatine is a novel melatonin (MT) receptor agonist at MT 1 and 2, serotonin receptor antagonist and an effective chronobiotic agent. The study was designed to evaluate the effects of agomelatine on body weight and food intake in restraint stress model in adult Swiss albino mice.

Methods: After the approval of Institutional Animal Ethics Committee, 40 male Swiss albino mice were randomly divided into four groups of 10 animals each; two were treatment groups which received $25 \mathrm{mg} / \mathrm{kg}$ (low dose) agomelatine, $50 \mathrm{mg} / \mathrm{kg}$ (high dose) agomelatine, standard group given trazodone and the control group administered the vehicle (1\% hydroxyethyl cellulose [HEC]) intraperitoneally for the last 14 days in the 3 weeks study period. Chronic restraint stress was given for 4 hrs per day for all groups starting from day 0 to 21 .

Results: Using paired t-test, both 12 hrs ( $\mathrm{p}=0.011)$ and $24 \mathrm{hrs}(\mathrm{p}<0.001)$ food intake in the high dose agomelatine group were significantly increased. Between groups using ANOVA test showed a statistically significant increase in food intake for this group when compared to the control group. Unlike the low dose agomelatine group $(\mathrm{p}=0.205)$, the mean body weight in the group treated with high dose agomelatine revealed a statistically significant rise compared to that of the control $(\mathrm{p}=0.001)$ in ANOVA test.

Conclusion: High dose agomelatine was effective in antagonizing the body weight lowering effect of restraint stress in addition to amelioration of reduced food intake. The study has potentially brought out the additional therapeutic benefit of agomelatine in improving the altered feeding and body weight changes when used in the treatment of the depression.

Keywords: Agomelatine, Body weight, Food intake, Satiety, Circadian rhythm.

(c) 2017 The Authors. Published by Innovare Academic Sciences Pvt Ltd. This is an open access article under the CC BY license (http://creativecommons. org/licenses/by/4. 0/) DOI: http://dx.doi.org/10.22159/ajpcr.2017.v10i9.19506

\section{INTRODUCTION}

Depression is a common illness worldwide, with an estimated 350 million people affected; constituting a major portion of mental health disorders [1]. A meta-analysis reported that the worldwide prevalence rate of depressive disorders in elderly population between $4.7 \%$ and $16 \%$ with a comparatively higher prevalence of $21.9 \%$ in India [2]. A study conducted in North India has shown that the prevalence of depression in the elderly was high even in a rural community with an estimate of $14.4 \%$ [3]. Hamilton Rating Scale for depression states that key features of depression include weight loss and reduced appetite [4]. Core symptoms according to diagnostic and statistical manual of mental disorders-V also includes eating too much/ little. Moreover a U-shaped association between body weight and depression has been described [5].

Agomelatine is a novel melatonin (MT) receptor agonist at MT1 and MT2 receptors, serotonin receptor 5-hydroxytryptamine $\left(5 \mathrm{HT}_{3} 2 \mathrm{C}\right)$ antagonist and an effective chronobiotic agent. It is found to be an effective antidepressant and an anxiolytic agent [6,7]. It has also been proved to improve sleep in a depressed patient by synchronizing the circadian rhythm [7].

Agomelatine, in addition to being a chronobiotic agent, has been shown to be effective in restraint stress induced depression in animal models [8]. Chronic restraint stress induces depressive-like behavior by increasing immobility time in the forced swimming test and cognitive deficit by producing memory impairment in the object recognition testing in animal models [9]. Restraint stress also affects the food intake and body weight of the animals [10]. There is a paucity of studies evaluating the effects of agomelatine on body weight and food intake in depression. Therefore the study was designed to evaluate the effects of agomelatine on body weight and food intake in restraint stress (immobility induced) model in adult Swiss albino mice.

\section{METHODS}

The Institutional Animal Ethics Committee approved the protocol of the study (Approval number: 241/2014/IAEC). Adult, male Swiss albino mice weighing 25-40 g and aged 5-10 months were housed in constant ambient temperature under 12 hrs light, 12 hrs dark cycle. They were group housed as 3-4 mice per cage in polypropylene cages. Pellet diet and tap water were provided ad libitum. The animals were randomly allocated into four groups of 10 mice per group.

- Group 1 - (treatment Group 1) low dose agomelatine was administered - $25 \mathrm{mg} / \mathrm{kg} /$ day

- Group 2 - (treatment Group 2) high dose agomelatine was administered - $50 \mathrm{mg} / \mathrm{kg} /$ day

- Group 3 - (standard group) trazodone $10 \mathrm{mg} / \mathrm{kg} /$ day

- Group 4 - control group given 1\% HEC.

The vehicle HEC is cellulose derived hydrophilic polymer [11]. The test, standard and vehicle (1\% HEC) were given intraperitoneally (single dose per day) to the animals in the respective groups for the last 14 days in the 3 weeks period. Chronic restraint stress was given for $4 \mathrm{hrs}$ per day starting from day 0 to day 21 for all groups.

The body weight and food intake (both 24 hrs and 12 hrs dark phase) were measured at $0,7,14,21$ days in each group [12]. After acclimatizing to individual housing for $12 \mathrm{hrs}$, food intake namely the $24 \mathrm{hrs}$ and $12 \mathrm{hrs}$ dark phase food intake were measured by keeping pre-weighed amount of food in each of the cages, in which the animals 
were individually housed and measuring the left-over food at the end of light phase (12 hrs) and dark phase (12 hrs). An accurate measurement was made by returning the spilled food at the bottom of the cage before measuring the weight of left-over food. Each animal was weighed and the body weight was recorded before the measurement of food intake on $0,7,14$, and 21 days (Fig. 1).

\section{RESULTS}

All data ware entered into excel and analyzed using SPSS software version 19. At baseline, a one way ANOVA test done showed that there was no significant difference in either the body weight $(\mathrm{p}=0.08)$ or the food intake, both the $12 \mathrm{hrs}(\mathrm{p}=0.53)$ and $24 \mathrm{hrs}(\mathrm{p}=0.05)$ in the four groups of animals indicating that there was no significant difference at baseline among the animals which were randomized to the four groups.

The food intake, both $12 \mathrm{hrs}$ and $24 \mathrm{hrs}$ in both the treatment groups (low dose and high dose) as well as the standard trazodone group showed an increase when compared between the $7^{\text {th }}$ day intake and $21^{\text {st }}$ day intake demonstrating the effects of drug treatment (Figs. 2 and 3). Using paired t-test, both $12 \mathrm{hrs}(\mathrm{p}=0.011)$ and $24 \mathrm{hrs}(\mathrm{p}<0.001)$ food intake in the high dose agomelatine group were found to be significantly increased. In addition, between groups analyses using ANOVA test showed a statistically significant increased food intake only in group 2 treated with high dose agomelatine when compared to control, but not the other two groups (Table 1).

A paired t-test done at the end of 7 days showed that there was a statistically significant reduction in weight in Groups 1-3 (Fig. 4) which were subsequently treated with low dose agomelatine, high dose agomelatine and trazodone respectively. The reduction in weight was induced as a result of the chronic restraint stress in this animal model.

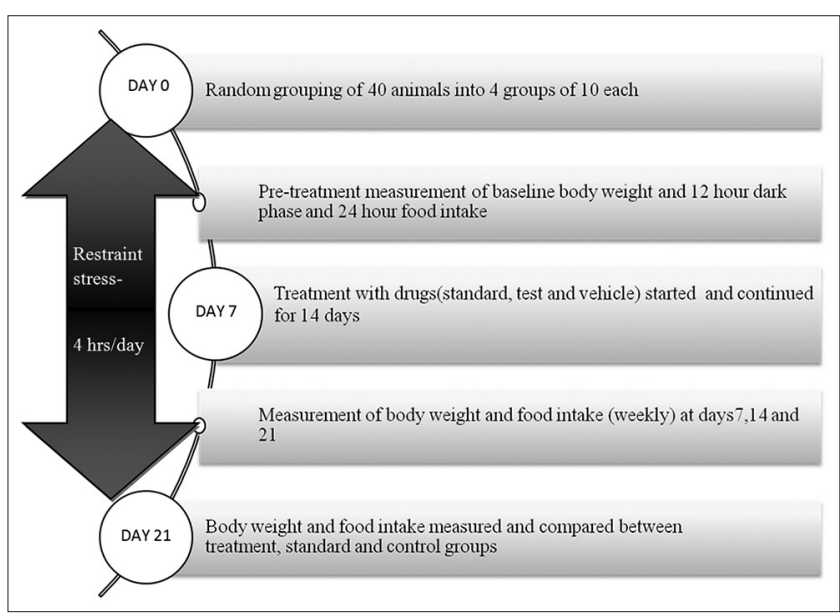

Fig. 1: Methodology of the study

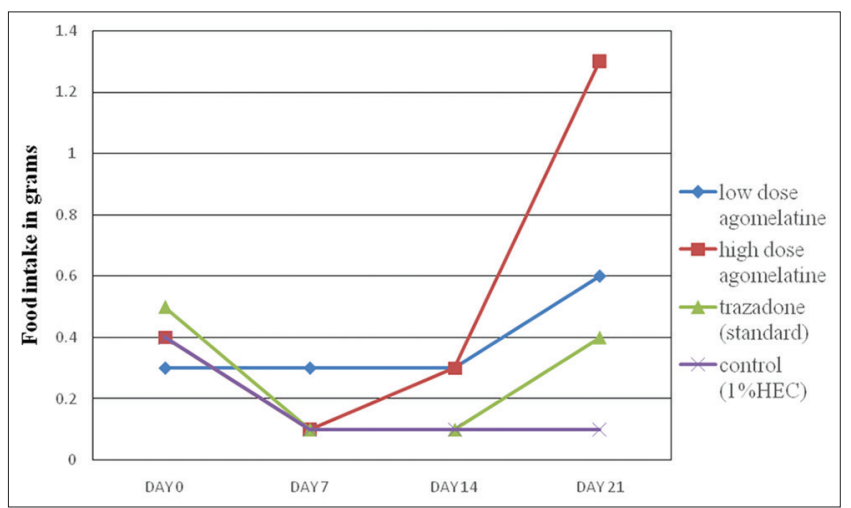

Fig. 2: Comparison of $12 \mathrm{hrs}$ mean food intake among groups
A paired t-test between the body weight measurements on day 7 and day 21 was done to detect a statistically significant change in mean body weight when agomelatine/trazodone was given along with the restraint stress. The low dose agomelatine group showed a statistically significant reduction in body weight $(\mathrm{p}=0.026)$. However, using ANOVA test, the changes in mean body weight were not significant in the groups which were treated with low dose agomelatine or trazodone compared to the control group (Fig. 4 and Table 1). The rise in mean body weight in the group treated with high dose agomelatine $(\mathrm{p}=0.001)$ when compared to control, was statistically significant indicating that agomelatine at $50 \mathrm{mg} / \mathrm{kg}$ was effective in antagonizing the body weight lowering effect of restraint stress. This was also mirrored in the statistical analysis of food intake between the three groups done at the end of 21 days (Table 1 ).

\section{DISCUSSION}

Agomelatine is a MT receptor agonist, serotonin receptor 5HT2C antagonist and an effective chronobiotic agent. It has been found to be an effective antidepressant and an anxiolytic [6]. Agomelatine synchronizes the circadian rhythm and also ameliorates stress-related changes like depressive symptoms by modulating the cytoskeleton micro tubular dynamics and synaptic markers such as brain derived neurotrophic factor in amygdala and hippocampus $[13,14]$. Agomelatine antagonizes serotonin $2 \mathrm{C}$ receptor which is an important mediator of satiety [15]. It is well established from studies conducted earlier that restraint stress model in mice induces depression such as state in addition to the reduction in body weight and food intake by altered expression of food related genes like decreased ghrelin and increased Pro-opiomelanocortin $[16,17]$.

A statistically significant increase in food intake was demonstrated only in the group treated with high dose agomelatine when compared

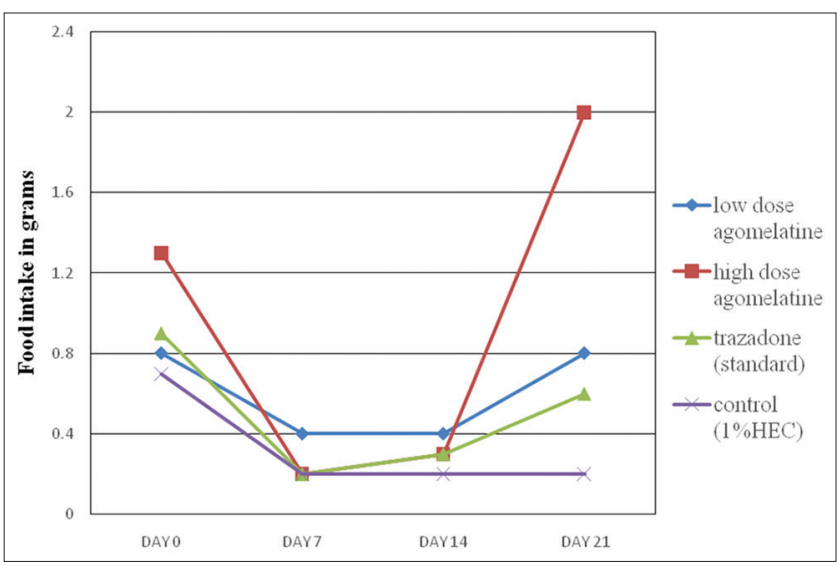

Fig. 3: Comparison of 24 hrs mean food intake among groups

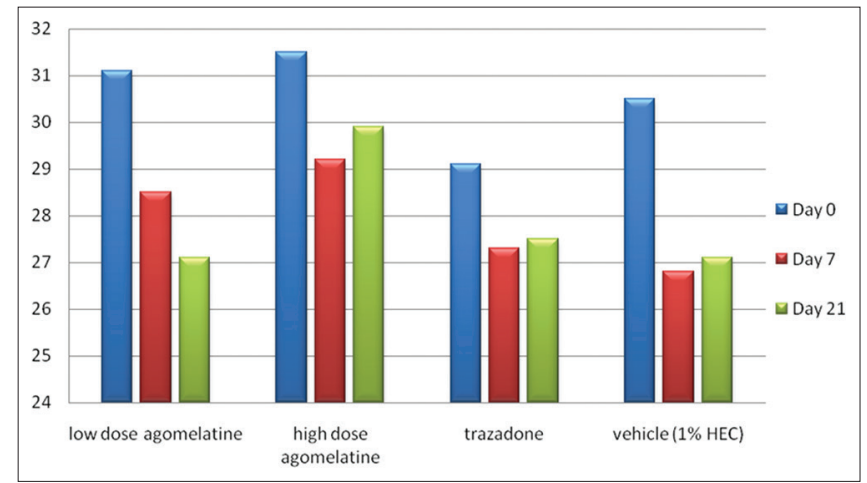

Fig. 4: Comparison of body weight (grams) among groups 
Table 1: Between-groups analysis using one-way ANOVA

\begin{tabular}{|c|c|c|c|c|c|c|}
\hline \multirow[t]{2}{*}{ Groups } & \multicolumn{2}{|c|}{12 hrs food intake } & \multicolumn{2}{|c|}{24 hrs food intake } & \multicolumn{2}{|c|}{ Body weight } \\
\hline & F statistic & p value & F statistic & p value & F statistic & p value \\
\hline Group 1 (low dose agomelatine) & 7.091 & 0.073 & 7.606 & 0.140 & 10.051 & 0.205 \\
\hline Group 3 (trazodone) & & 0.275 & & 0.321 & & 0.176 \\
\hline
\end{tabular}

to control, but not in groups which were treated with low dose agomelatine and trazodone (Table 1). This has confirmed that though there was an increased food intake evident in all the three groups, it was the high dose agomelatine which has significantly increased the food intake higher than that of the control group at the end of the treatment period, thereby effectively neutralizing the effect of restraint stress on the body weight. This also explains why the high dose agomelatine group had mean body weight significantly higher compared to that of control in contrary to the low dose agomelatine and trazodone groups.

The reasons for the effects of agomelatine on body weight and food intake are twofold. The first being the antidepressant activity of the drug, responsible for the therapeutic effect on the animals in depression-like state induced by chronic restraint stress which secondarily has led to the changes in body weight and improved food intake in the stressed animals. Second, this can be explained as due to the agomelatine's action at the serotonin receptor 5HT2C, which is a serotonin receptor subtype that is involved in normal satiation following a meal. Agomelatine antagonizes the 5HT2C receptor, and thereby it interferes with the satiety, increasing the food intake in the treated animals. The effect was well manifested in the group treated with high dose agomelatine. In contrast, the low dose agomelatine group did not show a similar response which probably could have been due to inadequate dosage to demonstrate such effects on weight and food intake. Thus, this study has delineated the effects of agomelatine on food intake and body weight when used as an antidepressant.

\section{CONCLUSION}

This study highlights the additional therapeutic benefit of agomelatine in the treatment of depression as it improves the altered feeding and body weight changes. The study clearly indicates the role of agomelatine in normalizing food intake and body weight in depressed patients with weight loss from poor feeding. These beneficial effects can be explained by its synchronizing action on circadian rhythm and antagonism of 5HT2C receptor, an important mediator of satiety.

\section{REFERENCES}

1. Reddy MS. Depression - The global crisis. Indian J Psychol Med 2012;34(3):201-3

2. Barua A, Ghosh MK, Kar N, Basilio MA. Prevalence of depressive disorders in the elderly. Ann Saudi Med 2011;31:620-4.

3. Pilania M, Bairwa M, Khurana H, Kumar N. Prevalence and predictors of depression in community-dwelling elderly in rural Haryana, India. Indian J Community Med 2017;42(1):13-8.

4. Worboys M. The Hamilton rating scale for depression: The making of a 'gold standard' and the unmaking of a chronic illness, 1960-1980. Chronic Illn 2013;9(3):202-19.

5. de Wit LM, van Straten A, van Herten M, Penninx BW, Cuijpers P. Depression and body mass index, a u-shaped association. BMC Public Health 2009;9:14.

6. Guaiana G, Gupta S, Chiodo D, Davies SJ, Haederle K, Koesters M. Agomelatine versus other antidepressive agents for major depression. Cochrane Database Syst Rev 2013;12:CD008851.

7. Liu J, Clough SJ, Hutchinson AJ, Adamah-Biassi EB, Popovska-Gorevski M, Dubocovich ML. MT1 and MT2 melatonin receptors: A therapeutic perspective. Annu Rev Pharmacol Toxicol 2016;56:361-83.

8. Reagan LP, Reznikov LR, Evans AN, Gabriel C, Mocaër E, Fadel JR. The antidepressant agomelatine inhibits stress-mediated changes in amino acid efflux in the rat hippocampus and amygdala. Brain Res 2012;1466:91-8

9. Budni J, Zomkowski AD, Engel D, Santos DB, dos Santos AA, Moretti $\mathrm{M}$, et al. Folic acid prevents depressive-like behavior and hippocampal antioxidant imbalance induced by restraint stress in mice. Exp Neurol 2013;240:112-21.

10. Jiang SZ, Eiden LE. Activation of the HPA axis and depression of feeding behavior induced by restraint stress are separately regulated by PACA Pergic neurotransmission in the mouse. Stress 2016;19(4):374-82.

11. Arafat M. Approaches to achieve an oral controlled release drug delivery system using polymers: A recent review. Int J Pharm Pharm Sci 2015;7(7):16-21.

12. Shanmugapriya S, Senthurselvi R, Bhuvaneswari K. A study comparing the anorectic activity of hot and cold aqueous extracts of Dolichos biflorus Linn. Seeds in freely feeding rats. Int J Pharm Pharm 2016;8(7):336-9.

13. Ladurelle N, Gabriel C, Viggiano A, Mocaër E, Baulieu EE, Bianchi M. Agomelatine (S20098) modulates the expression of cytoskeletal microtubular proteins, synaptic markers and BDNF in the rat hippocampus, amygdala and PFC. Psychopharmacology (Berl) 2012;221(3):493-509.

14. Conboy L, Tanrikut C, Zoladz PR, Campbell AM, Park CR, Gabriel C, et al. The antidepressant agomelatine blocks the adverse effects of stress on memory and enables spatial learning to rapidly increase neural cell adhesion molecule (NCAM) expression in the hippocampus of rats. Int J Neuropsychopharmacol 2009;12(3):329-41.

15. de Bodinat C, Guardiola-Lemaitre B, Mocaër E, Renard P, Muñoz C, Millan MJ. Agomelatine, the first melatonergic antidepressant: Discovery, characterization and development. Nat Rev Drug Discov 2010;9(8):628-42

16. Haque Z, Akbar N, Yasmin F, Haleem MA, Haleem DJ. Inhibition of immobilization stress-induced anorexia, behavioral deficits, and plasma corticosterone secretion by injected leptin in rats. Stress 2013;16(3):353-62.

17. Jeong JY, Lee DH, Kang SS. Effects of chronic restraint stress on body weight, food intake, and hypothalamic gene expressions in mice. Endocrinol Metab (Seoul) 2013;28(4):288-96. 